\title{
Spatio-Temporal Nonrigid Registration for Ultrasound Cardiac Motion Estimation
}

\author{
María J. Ledesma-Carbayo*, Member, IEEE, Jan Kybic, Member, IEEE, Manuel Desco, \\ Andrés Santos, Senior Member, IEEE, Michael Sühling, Student Member, IEEE, Patrick Hunziker, and \\ Michael Unser, Fellow, IEEE
}

\begin{abstract}
We propose a new spatio-temporal elastic registration algorithm for motion reconstruction from a series of images. The specific application is to estimate displacement fields from two-dimensional ultrasound sequences of the heart. The basic idea is to find a spatio-temporal deformation field that effectively compensates for the motion by minimizing a difference with respect to a reference frame. The key feature of our method is the use of a semi-local spatio-temporal parametric model for the deformation using splines, and the reformulation of the registration task as a global optimization problem. The scale of the spline model controls the smoothness of the displacement field. Our algorithm uses a multiresolution optimization strategy to obtain a higher speed and robustness.

We evaluated the accuracy of our algorithm using a synthetic sequence generated with an ultrasound simulation package, together with a realistic cardiac motion model. We compared our new global multiframe approach with a previous method based on pairwise registration of consecutive frames to demonstrate the benefits of introducing temporal consistency. Finally, we applied the algorithm to the regional analysis of the left ventricle. Displacement and strain parameters were evaluated showing significant differences between the normal and pathological segments, thereby illustrating the clinical applicability of our method.
\end{abstract}

Index Terms-Cardiac motion, elastic registration, parametric models, splines, temporal models.

\section{INTRODUCTION}

$\mathbf{T}$ HE ESTIMATION of cardiac motion constitutes an important aid for the quantification of the elasticity and contractility of the myocardium. Localized regions exhibiting

Manuscript received May 9, 2005; revised May 17, 2005. This work was supported in part by the Swiss Science Foundation, the Spanish Health Ministry under Grant 3200-059517.99/1 (research project PI041495 and Red Temática IM3 G03/185) and in part by the Czech Ministry of Education under Project MSM6840770012. The Associate Editor responsible for coordination the review of this paper and recommending its publication was M. Viergever. Asterisk indicates corresponding author.

*M. J. Ledesma-Carbayo is with ETSI Telecomunicación, Universidad Politécnica de Madrid, Ciudad Universitaria s/n, E-28040 Madrid, Spain (e-mail: mledesma@die.upm.es).

J. Kybic was with Biomedical Imaging Group, EPFL, Switzerland. He is now with Center for Machine Perception, Czech Technical University, Prague, Czech Republic (e-mail: kybic@fel.cvut.cz).

M. Desco is with Medicina y Cirugía Experimental, Hospital G. U. Gregorio Marañón, Dr. Esquerdo 46, E-28007 Madrid, Spain (e-mail: desco@mce.hggm.es).

A. Santos is with ETSI Telecomunicación, Universidad Politécnica de Madrid, Ciudad Universitaria s/n, E-28040 Madrid, Spain (e-mail: andres@die.upm.es).

M. Sühling and M. Unser are with the Biomedical Imaging Group, Swiss Federal Institute of Technology Lausanne (EPFL), CH-1015 Lausanne, Switzerland (e-mail: michael.suehling@epfl.ch; michael.unser@epfl.ch).

P. Hunziker is with the University Hospital Basel, CH-4031 Basel, Switzerland (e-mail: PHunziker@uhbs.ch).

Digital Object Identifier 10.1109/TMI.2005.852050 movement abnormalities are indicative of the existence of ischemic segments, which are caused by insufficient tissue microcirculation. Currently, the reference modality for motion estimation is tagged magnetic resonance (MR) imaging, which allows us to obtain cardiac displacement fields and derived parameters, such as the myocardial strain, with high accuracy [1]-[4]. Most approaches using magnetic resonance imaging (MRI), single photon emission comuted tomography (SPECT), and computed tomography (CT) are based on deformable and mechanical models, and they require a presegmentation step [2], [3], [5]-[7]. Other methods use energy-based registration [4], [8], [9] and optical flow techniques [10] to compute the displacement of the myocardium.

Sequence alignment and registration methods for motion detection have been investigated in computer vision [11]-[13]. Registration methods have been used in cardiac imaging; they are usually applied to data acquired at the same time point in the cardiac cycle, with the aim of achieving either multimodal integration [14] or to compensate for small misalignments [15]. Image registration has also been successfully applied for estimating cardiac motion in tagged MR data [4], [6], [16]-[19]. Some of these methods impose spline temporal models to assure temporal consistency and better motion tracking [4], [11], [16], [17].

Our work concentrates on two-dimensional (2-D) echocardiography, as it is ubiquitous, and is the most widely used imaging method to assess cardiac function. The techniques proposed for cardiac motion recovery in other modalities cannot be applied directly, because of the especific features of echocardiographic data.

1) The signal-to-noise ratio (SNR) is relatively low and dependent on the angle of incidence and depth. Signal dropouts may appear because of "shadowing," even though the development of recent image acquisition techniques, such as second harmonic imaging, allow for better performance in this respect.

2) The complex three-dimensional motion of the heart results in a partially decorrelated speckle when 2-D sequences are analyzed [20], [21], therefore making interframe relation weaker. Similar effects are observed for out-of-plane motion, which may cause intracardiac structures (for example papillary muscles or valve cordae) entering and leaving the view plane, a limitation shared by other 2-D modalities.

Different approaches have been proposed for motion recovery from 2-D echocardiagraphic sequences. The most popular is myocardial border segmentation using deformable models [22]-[25]. Some of these methods try to overcome 
the intrinsic complexity of the data by introducing a priori knowledge by modeling a statistical representation of the possible motions and shapes [24], [25]. However these techniques estimate the cardiac motion considering the myocardial borders only; this can lead to inaccurate motion estimations when the movement is parallel to the border. An additional problem is that the borders are usually not well defined in echocardiographic data. Another approach is to use optical flow methods to compute local myocardial movements [26]-[31]. Specific designs for echocardiographic data consider the Rayleigh statistics of the signal in the process [29]. Both differential and block matching techniques add mechanical, spatial or temporal constraints to overcome the well-known aperture problem with solutions adapted for echocardiography [26], [27], [30], [31]. The third approach with promising results is to obtain myocardial motion and deformation using speckle tracking [32] and elastographic techniques [33]-[35]. These methods are based on the processing of the RF signal to obtain the displacement of one or several consecutive lines of response, using correlation and phase shift techniques.

In this paper, we propose using a nonrigid parametric motion estimation algorithm developed to overcome some of the underlying problems inherent to echocardiographic image tracking. Our approach is global, in the sense that it considers all the frames in the sequence together, and that it tries to find the most globally plausible dense spatio-temporal motion field. The deformation field is represented using a parametric model based on B-spline basis functions.

The algorithm does not require any preliminary segmentation, which would be particularly difficult in the case of cardiac ultrasound images. The spatio-temporal parametric model together with a multiresolution optimization strategy provide a good framework for tracking both global shape and texture. The multiresolution approach increases speed and tolerance to noise.

The underlying assumption for our approach is that the echo signal is due to the presence of strong scatterers in the tissue that produce large and bright speckles [36]. While these scatterers move in-plane, they produce a signal component with stable and visible texture pattern whose displacement is directly linked with the in-plane cardiac motion. On the other hand, the out-of-plane movement of the scatterers produces a speckle component decorrelated with time [20], [21]. Our algorithm is designed to lock on the temporally coherent part of the signal, while suppressing the second component as much as possible. This is achieved by imposing temporal and spatial smoothness constraints on the deformation field.

The paper is organized as follows. In Section II, we present our method in detail, covering all the methodological aspects. In Section III, we evaluate the algorithm using simulated sequences derived from a realistic cardiac motion model. In Section IV, we present the results obtained from a clinical trial in which regional cardiac analysis of the left ventricle was performed in a population of patients and in healthy controls.

\section{Spatio-Temporal Registration}

\section{A. Problem Definition}

Let us consider an image sequence $f(t, \mathbf{x})$ with $t=$ $0, \ldots, T-1$ and $\mathbf{x} \equiv\left(x_{1}, x_{2}\right) \in \mathbf{I}$, where $f(t, \mathbf{x})$ is the intensity at time $t$ and position $\mathbf{x}$. Our goal is to find a dense displacement field over the whole sequence; to this end, we introduce the deformation function, $\mathbf{g}(t, \mathbf{x})$, which represents the position at time $t$ of a point that was at position $\mathbf{x}$ at time $t=0$, i.e., the so-called Lagrangian representation. In other words, we are using the first frame as a spatial reference, implying $\mathbf{g}(0, \mathbf{x})=\mathbf{x}$.

\section{B. Consecutive Registration}

This registration method is described in [37], and is based on the registration of consecutive pairs of images obtained from the sequence, using an algorithm derived from [38]. This approach calculates the interframe displacement fields $\mathbf{g}_{t}(\mathbf{x})$. The total deformation field, $\mathbf{g}(t, \mathbf{x})$, is then obtained from the contribution of the partial fields.

Registration is performed twice, in the forward and backward directions, to minimize any error accumulation, and the mean of the two displacements is used as the final result. We have also imposed a periodicity on the measurements, as the sequence encompasses a complete cycle.

In the remainder of the paper, we shall denote this method as "consecutive elastic registration," or C-Reg.

\section{Spatio-Temporal Registration}

In contrast to the consecutive registration method, the new algorithm presented in this article works globally on all the images of the sequence simultaneously. It searches for a spatio-temporal deformation field, $\mathbf{g}(t, \mathbf{x})$, expressed by a parametric B-spline model. By applying this field to warp the original sequence, $f$, we obtain a motion-corrected sequence, $f_{w}(t, \mathbf{x})=f(t, \mathbf{g}(t, \mathbf{x}))$, that should resemble the reference frame as much as possible. In other words, $f_{w}(t, \mathbf{x})$ should appear stationary. The key features of the algorithm are the similarity criterion (Section II-D), and the spatio-temporal deformation model (Section II-F).

Fig. 1 shows an example of the spatio-temporal registration process and the results obtained. The upper part of Fig. 1 shows three images from the original sequence covering the entire cardiac cycle. The lower part shows the corresponding images from the warped (i.e., motion-corrected) sequence.

\section{Optimization Criterion}

Our registration procedure seeks a minimum value, $\mathrm{g}=\operatorname{argmin}_{\mathbf{g} \in G} E(\mathrm{~g})$, for a criterion, $E$, which is defined as the mean value obtained from the entire sequence of an image similarity criterion, $E_{t}$

$$
\begin{aligned}
E & =\frac{1}{T} \sum_{t=0}^{T-1} E_{t} \\
E_{t} & =\frac{1}{N_{I}} \sum_{\mathbf{i} \in \mathbf{I}}(f(t, \mathbf{g}(t, \mathbf{i}))-f(0, \mathbf{i}))^{2}
\end{aligned}
$$

where $T$ is the total number of images in the sequence, $\mathbf{I}$ is the set of coordinates specifying the spatial region of interest, and $N_{I}$ is the corresponding number of pixels. The image criterion, $E_{t}$, is the average of the square of the differences with respect 


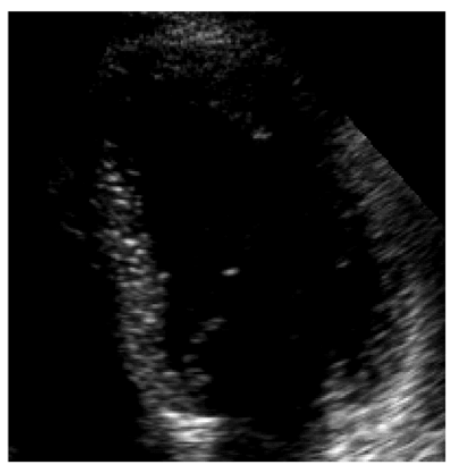

End of Diastole (Reference Frame)

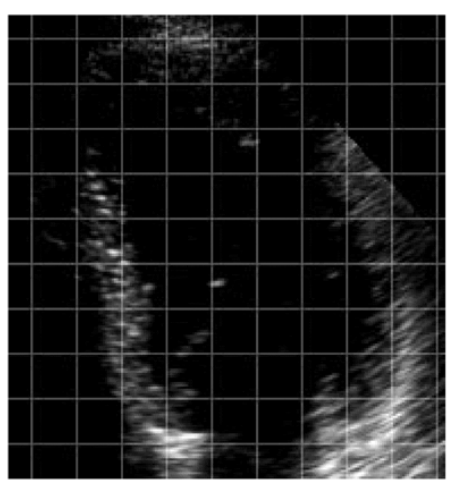

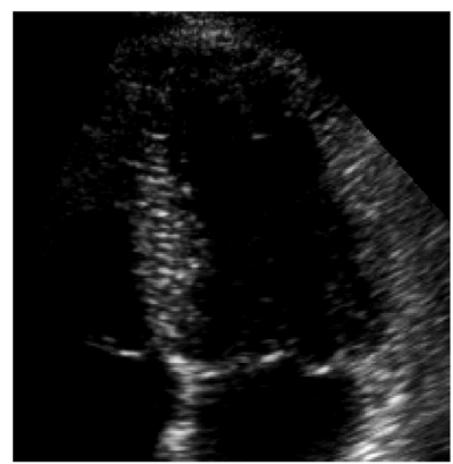

End of Systole

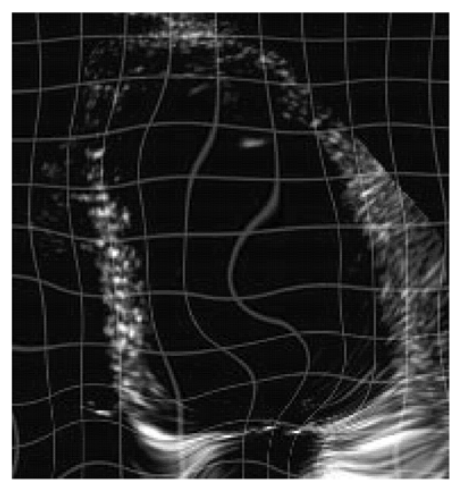

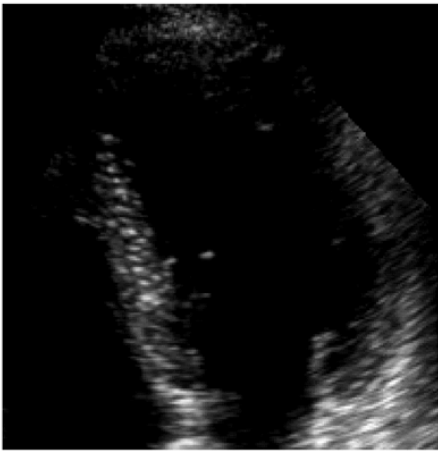

Mid Diastole

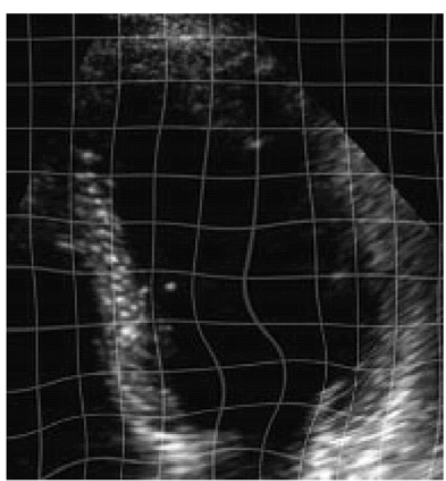

Fig. 1. The spatio-temporal registration process, showing the original sequence images (top), and the corresponding images in the warped sequence (bottom), which tend to appear stationary.

to the reference image at time $t=0$ (and is equivalent to the sum of squared differences, SSD).

We chose to use the SSD criterion because of its simplicity, fast computation time, and smoothness of the resulting criterion space. We extended this criterion to the temporal dimension, and observed that this criterion performed well, even in the presence of noise and partially decorrelated speckle. We selected the end-diastolic frame as the reference frame, because it is easily identified in ultrasound sequences from the R-wave of the electrocardiogram (ECG).

\section{E. Interpolation}

It is necessary to have a continuous version of $f$ to be able to calculate the warped sequence, $f(g(\cdot))$, by interpolation, as well as to be able to evaluate the criterion derivatives. To this end, we chose to represent $f$ using a 2-D spline interpolation

$$
f(t, \mathbf{x})=\sum_{\mathbf{i} \in \mathbb{Z}^{2}} b_{\mathbf{i}, t} \beta^{n}(\mathbf{x}-\mathbf{i}) \quad \mathbf{x} \in \mathbf{I} \quad t \in 0,1, \ldots, T-1
$$

where $\beta^{n}(\mathbf{x})$ is the tensor product of centered uniform B-splines of degree $n$. (Note, $n=3$ was used in all our experiments). The coefficients, $b_{\mathbf{i}, t}$, were obtained from the pixel values, $f(\mathbf{i})$, using filtering [39]. The spline model has the advantage of good accuracy, low computational complexity, and allows for the possibility of evaluating spatial derivatives analytically.

\section{F. Spatio-Temporal Model}

The deformation function, $\mathbf{g}(t, \mathbf{x})$, is represented by a linear model, which is separable in time and space, with parameters, $\mathbf{d}_{\mathbf{j}, l}$

$$
\mathrm{g}(t, \mathbf{x})=\mathbf{x}+\sum_{l \in L} \sum_{\mathbf{j} \in \mathbf{J}} \mathbf{d}_{\mathbf{j}, l} \phi_{\mathbf{j}}(\mathbf{x}) \psi_{l}(t)
$$

where $\mathbf{J} \subset \mathbb{Z}^{2}$ and $L \subset \mathbb{Z}$ define the set of spatial and temporal parameter indices. The parameter $\phi_{\mathbf{j}}(\mathbf{x})$ defines the basis functions in the spatial direction, and is responsible for the spatial smoothness, and $\psi_{l}(t)$ are the time-axis basis functions that impose the temporal coherence of the deformation. As shown in [11], [38], and [40], B-splines constitute a good choice for the spatial basis functions, $\phi_{\mathbf{j}}$. We also used B-splines for the temporal basis functions, $\psi_{l},[4]$, [11], [16], [17], because of their computational simplicity, good approximation properties, and implicit smoothness (minimum curvature property). We found that temporal B-splines performed at least as well as harmonic functions (as used in [1], [2], and [5]) in terms of registration accuracy, with the advantage that the criterion minimization was easier, thanks to their compact support [41]. Specifically, we used the following basis functions:

$$
\phi_{\mathbf{j}}(\mathbf{x})=\beta^{n}\left(x_{1} / h-j_{1}\right) \cdot \beta^{n}\left(x_{2} / h-j_{2}\right)
$$

where

$$
\mathbf{j}=\left(j_{1}, j_{2}\right)
$$

and

$$
\psi_{l}(t)=\beta^{n}(t / s-l) .
$$



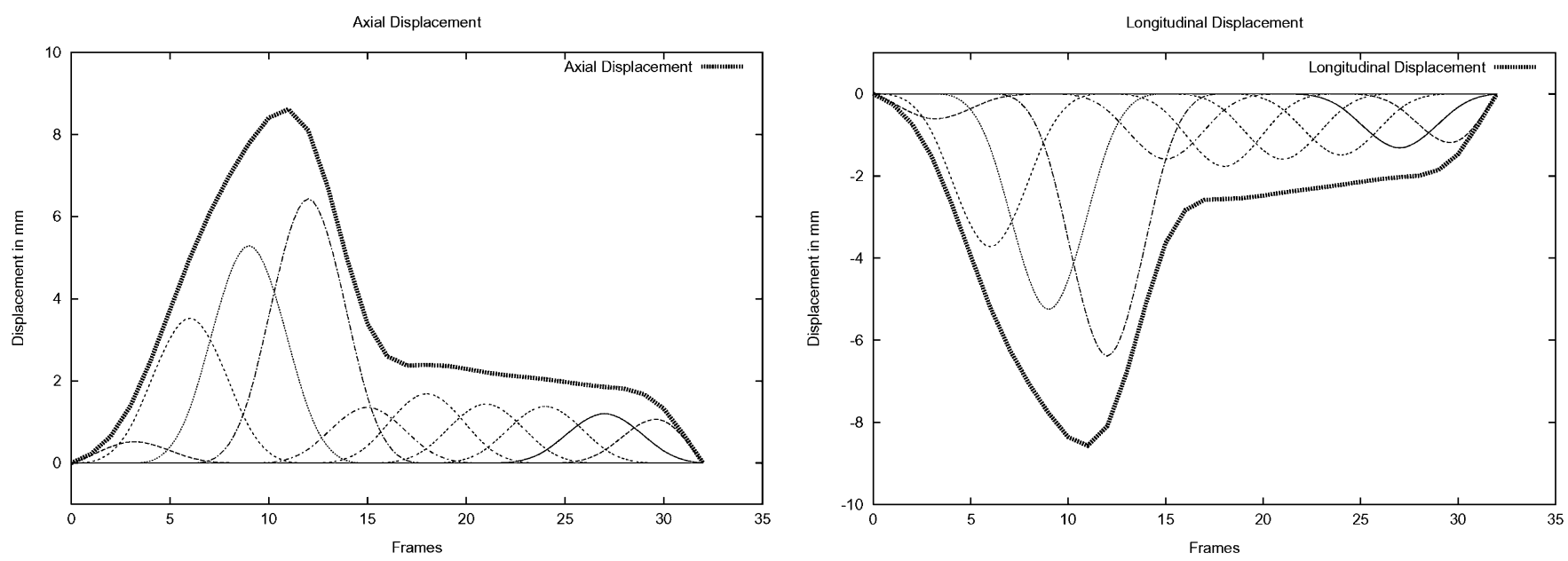

Fig. 2. The B-splines temporal model. The axial and longitudinal displacement of a point in the myocardium is defined by the temporal B-spline basis functions. The individual scaled basis functions are shown, along with their sum, the total trajectory.

The basis functions, $\phi_{\mathbf{j}}(\mathbf{x})$, were placed on a uniform rectangular spatial grid, and the $\psi_{l}(t)$ were placed at regularly spaced time intervals. The scale parameters $h$ (space) and $s$ (time) govern the knot spacing and, therefore, the total number of parameters. These parameters also control the rigidity of the solution. Typically, we used quadratic B-splines, $\beta^{2}$, as $\phi$, and cubic B-splines, $\beta^{3}$, as $\psi$, with $h=32$ and $s=5$. In Section III-B2 we analyze the influence of the knot spacings in more detail. We found that, using cubic B-splines in the spatial direction did not improve the accuracy significantly and was not worth the additional computational effort.

\section{G. Motion Field Constraints}

The motion model of (5) can be further constrained by using a priori knowledge of the motion field. This increases the robustness of the registration process by taking out superfluous degrees of freedom. First, we know that the displacement at the reference frame $(t=0)$ must be zero. This removes one degree of freedom from our problem, and leads to a modified basis function set that only generates displacements satisfying this constraint

$$
\psi_{l}^{\prime}(t)=\psi_{l}(t)-\frac{\psi_{l}(0) \psi_{l_{0}}(t)}{\psi_{l_{0}}(0)} .
$$

Similarly, if our sequence contains a full cycle, then we set the reference frame to $t=0$, and we impose $\mathrm{g}(T)=0$, leading to a cyclic set of basis functions defined by

$$
\psi_{l}^{\prime \prime}(t)=\psi_{l}^{\prime}(t)-\frac{\psi_{l}^{\prime}(T) \psi_{l_{\max }}^{\prime}(t)}{\psi_{l_{\max }}^{\prime}(T)} .
$$

Fig. 2 shows how these modified basis functions define the axial and longitudinal displacement for a point in the myocardium. It depicts the individual basis functions scaled using proper coefficients, as well as the overall trajectory $\mathbf{g}\left(t, \mathbf{x}_{0}\right)$.

\section{H. Multiresolution and Optimization Strategy}

The solution to our registration problem is a deformation field, $\mathbf{g}$, that minimizes the criterion, $E(\mathrm{~g})$. This is found by using a multidimensional optimization algorithm acting on the parameters $\mathbf{d}_{\mathbf{j}, l}$. The required partial derivatives of $E$ can be calculated explicitly

$$
\begin{aligned}
\frac{\partial E}{\partial \mathbf{d}_{\mathbf{j}, l, m}}=\frac{1}{N_{I} T} & \sum_{k=0}^{T-1} \sum_{\mathbf{i} \in \mathbf{I}} 2(f(t, \mathbf{g}(k, \mathbf{i})) \\
& -f(0, \mathbf{i}))\left.\frac{\partial f(t, \mathbf{x})}{\partial x_{m}}\right|_{\mathbf{x}=\mathbf{g}(k, \mathbf{i})} \frac{\partial \mathbf{g}_{m}(k, \mathbf{i})}{\partial \mathbf{d}_{\mathbf{j}, l, m}}
\end{aligned}
$$

while the partial derivatives of $\mathbf{g}(t, \mathbf{x})$ as given by (4) and $f(t, \mathbf{x})$ as in (3) are

$$
\begin{aligned}
\frac{\partial \mathbf{g}_{m}(t, \mathbf{x})}{\partial \mathbf{d}_{\mathbf{j}, l, m}} & =\beta^{n}(t / s-l)\left(\beta^{n}\left(x_{1} / h-j_{1}\right) \cdot \beta^{n}\left(x_{2} / h-j_{2}\right)\right) \\
\frac{\partial f(t, \mathbf{x})}{\partial x_{m}} & =\left.\sum_{\mathbf{i} \in \mathbb{Z}^{2}} b_{\mathbf{i}, t} \beta^{\prime n}\left(x_{m}\right) \beta^{n}\left(x_{\lambda}-i_{\lambda}\right)\right|_{\lambda \neq m} .
\end{aligned}
$$

We used a gradient descent method with an automatic step-size update [38]. We applied a multiresolution optimization strategy that ensured a robust and efficient approach. A pyramid of progressively reduced versions of the original sequence was created by fitting the data using splines with coarser levels of resolution (a spatio-temporal wavelet-like pyramid) [42]. This pyramid was compatible with our sequence model (3), and was optimal in the $L_{2}$-sense. We also used multiresolution for the motion model, beginning with a coarsely defined deformation function, $\mathbf{g}$, with a few parameters, $\mathbf{d}_{\mathbf{j}, l}$, and then increasing the number of parameters until the finest representation of the model was achieved. After converging at a given level, the result was then used as an initial estimation for the ensuing, finer level. The projection onto the finer space was achieved using no approximations, thanks to the embedding properties of the underlying B-spline spaces.

To summarize, the optimization process proceeded in a coarse-to-fine fashion for both the image sequence and the motion field model. The optimization stopped when the changes in $\Delta \mathbf{d}$ were below a given a priori threshold, $\varepsilon$. The convergence speed depends on the number of parameters and the sequence size. In a typical $350 \times 230$ image size, with 35 frames, and parameters $h=32, s=5$, with 8 multiresolution levels and 

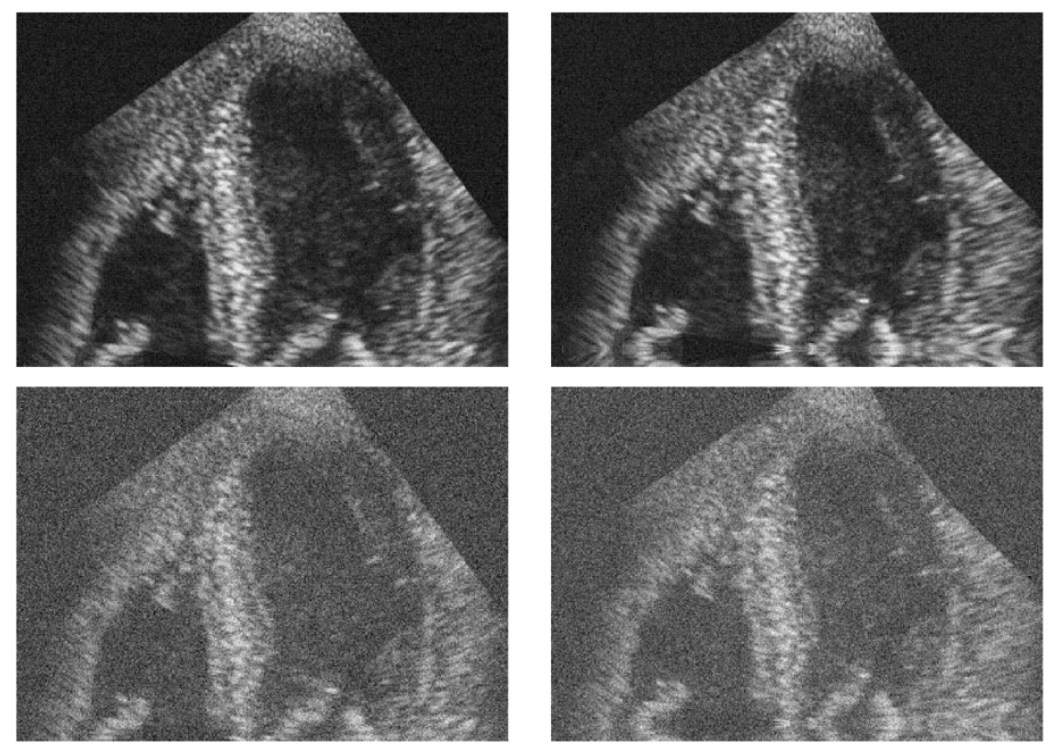

Fig. 3. First and tenth frames of simulated sequences $S_{1}$ (top) and $S_{2}$ (bottom).

a threshold of $\varepsilon=0.01$, the current version of the algorithm coded in Python needed about half an hour using a standard PC ( $\sim 20$ iterations for each multiresolution level). We expect a fivefold-to-tenfold reduction in computation time when the algorithm is completely recoded in C. We observed that the algorithm always converged to a sensible solution.

\section{EXPERIMENTS WITH SiMULATED DATA}

This section discusses evaluation experiments on simulated data. We analyzed the benefits of the temporal model and the influence of the different algorithm parameters. The use of simulated sequences allows us to quantify the accuracy of the reconstructed motion, which would not be possible to obtain with real data.

As the true cardiac motion was not available, we generated a realistic cardiac motion field, as described in Appendix A. The corresponding model was separable, and consisted of two components: an affine spatial component that simulated radial myocardial contraction or expansion, and a temporal component that modulated this movement in a realistic fashion throughout the cardiac cycle.

\section{A. Simulated Sequences}

We generated two different sets of simulated sequences using the model mentioned above. The first set was defined to explore the behavior of the algorithm under controlled noise. To generate this first set, we took one real, end-diastole apical view image, and deformed it according to the motion model (12). We corrupted the deformed images using different levels of additive Gaussian noise. We generated three sequences with increasing noise levels: $S_{0}$ (noiseless), $S_{1}$ (SNR $\sim 25 \mathrm{~dB}$ ), and $S_{2}$ (SNR $\sim 15 \mathrm{~dB}$ ). Fig. 3 shows the first and tenth frames of the $S_{1}$ and $S_{2}$ simulated sequences.

Second, we generated a sequence $S_{\text {us }}$ using the FIELD II ultrasound simulation package [43], [44]. This package provides an excellent framework to simulate ultrasound fields. It incorporates realistic transducer features, even though the latest ultrasound imaging acquisition technologies, such as second harmonic imaging or fusion imaging, are not included.

The main purpose of generating this sequence was to include more realistic ultrasonic features, while keeping known motion. The simulation of the ultrasound field was based on the computation of the spatial impulse response, including the excitation scheme (dynamic focusing and apodization). The images were generated from a map of independent scatterers with determined positions and amplitudes [45]. To generate our test sequence, we specified a typical cardiac transducer (3-MHz central frequency, 64-element phased array with Hanning apodization for both transmission and reception, and with single focus in transmission and multiple focusing in reception mode).

The phantom scatterer amplitudes and positions were generated from a sequence of scattering strength maps. These maps modeled the different densities and speed of sound in the different tissues [45]. We designed the first frame of the scattering map using a real end-diastole image as a template. The entire sequence of maps was then generated by deforming the first map according to the motion model (12). For each image, 200000 scatterers were generated using random positions, simulating a 1-cm-thick slice of the heart. The amplitude of the scatterers followed a Gaussian distribution that was determined by the scattering map value at each position. The final image was calculated by summing the responses of all the scatterers, which were specified by their positions and amplitudes [45]. We used 128 scanning lines to define the image sector, and the resultant image size was $359 \times 256$ pixels. Alone, this process would generate an unrealistically decorrelated sequence in time as we run independent random processes for each frame to select the scatterer positions. Therefore, we imposed some interframe correlation through the introduction of stable scatterers in the myocardium. These composed about $5 \%$ of the total number of scatterers in the myocardium, and their position in the tissue did not change. Therefore, the sequence, $S_{\text {us }}$, was weakly correlated in time, 

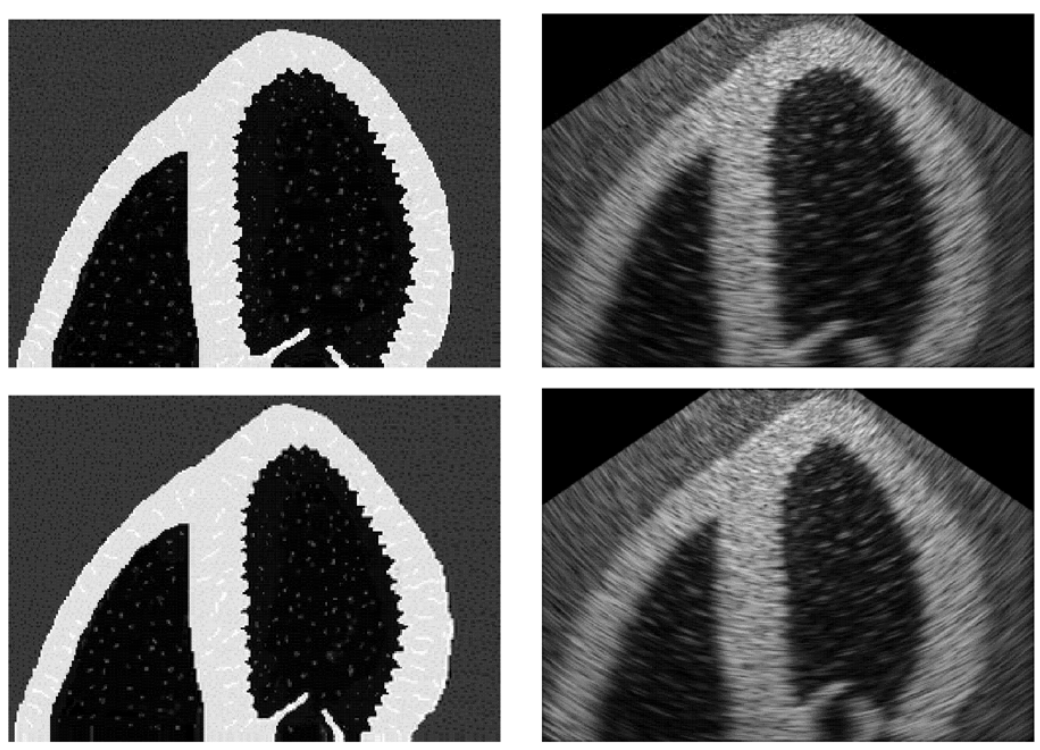

Fig. 4. The first and tenth frames of the scattering maps (left), and the corresponding ultrasound simulated images (right).

which permitted us to evaluate the performance of our method in an almost realistic setting. Fig. 4 shows the scatter map for the first and tenth frames, and the corresponding ultrasound simulated frames in $S_{\text {us }}$.

\section{B. Experiments and Results}

This section discusses a series of experiments carried out to evaluate the performance of the algorithm. We tested the influence of the various parameters involved, and the extent to which our spatio-temporal approach added robustness and accuracy to any motion estimation, in comparison to our previous approach [37]. We measured the accuracy of the motion estimation using a warping index [46], which was defined as the mean geometric error in pixels between the true and the recovered deformation, defined as $\varpi=\sqrt{\left(1 / N_{R} T\right) \sum_{k \in T} \sum_{\mathbf{i} \in R}\left\|\mathbf{g}(k, \mathbf{i})-\mathbf{g}_{0}(k, \mathbf{i})\right\|^{2}}$, where $R$ is the region of interest, the myocardium in our case, $N_{R}$ is the number of pixels in this region, and $T$ is the total number of images in the sequence. This index represents an overall measure of the local error.

The synthetic motion model (12) was deliberately expressed outside the spatio-temporal deformation space (4) searched by the algorithm to avoid a biased scenario. We calculated the minimum geometric error between the motion model and the best possible representation in the spatio-temporal search space (the projection of the cardiac motion model onto the deformation space). In the following sections, we will refer to this minimum error as the "ideal" error.

1) Robustness With Respect to Noise: The first experiment analyzed the effects of noise on our algorithm. The experiments were carried out on all four simulated sequences (Section III-A). Sequences $S_{0}$ to $S_{2}$ allowed for the isolation of the influence of noise, and the sequence $S_{\text {us }}$ allowed us to test the algorithm in a more realistic setting. We compared the method with our previous approach (C-Reg), which was briefly described in Section II-A. We used the same parameter settings for both algorithms: Spacing between knots was $h=32$ pixels, quadratic
TABLE I

WARPING INDEX (GEOMETRIC ERROR) IN PIXELS FOR THE FOUR TEST SEQUenCES Described IN SECTION III-A FOR THE New Algorithm (ST-Reg), AND THE PREVIOUS METHOD (C-Reg) [37]

\begin{tabular}{c||r|r|r|r}
\hline Sim & Ideal-C & C-Reg & Ideal-ST & ST-Reg \\
\hline$S_{0}$ & 0.168 & 0.534 & 0.347 & 0.631 \\
\hline$S_{1}$ & 0.168 & 0.647 & 0.347 & 0.662 \\
\hline$S_{2}$ & 0.168 & 1.629 & 0.347 & 0.706 \\
\hline$S_{u s}$ & 0.168 & 4.815 & 0.347 & 1.265 \\
\hline
\end{tabular}

B-splines were used to represent the deformation, and the stopping threshold was $\varepsilon=0.01$. For the spatio-temporal registration (denoted here by $S T$-Reg), we used a spacing between temporal knots of $s=5$ frames, and quadratic B-splines as the corresponding basis function. We also calculated the minimum possible approximation errors within the search space for both algorithms, denoted here as Ideal-C and Ideal-ST.

Table I shows the results of the warping index for both algorithms for the four test sequences. In the cases with least noise (i.e., sequences $S_{0}$ and $S_{1}$ ), the consecutive registration algorithm performed marginally better, because of its less constrained motion model. In the other cases $\left(S_{2}\right.$ and $\left.S_{\text {us }}\right)$, the new spatio-temporal algorithm was significantly better, yielding a mean geometric error for the realistic sequence, $S_{\mathrm{us}}$, of 1.265 pixels, which corresponds to $5 \%$ of the maximum displacement. Note that the ideal values were not attained. However, the difference was small - within the range of half a pixel for the noiseless case $S_{0}$.

Fig. 5 shows the true axial and longitudinal components of the displacement in the sequences, the displacements found by the algorithms, and the best achievable (ideal) result within the frame of the imposed motion model. The curves are drawn for a middle-septum point in sequences $S_{2}$ and $S_{\mathrm{us}}$. One can see the inconsistent and underestimated results of the consecutive algorithm. This underestimation is due to the accumulation of the estimated consecutive displacements and to the regularization in- 

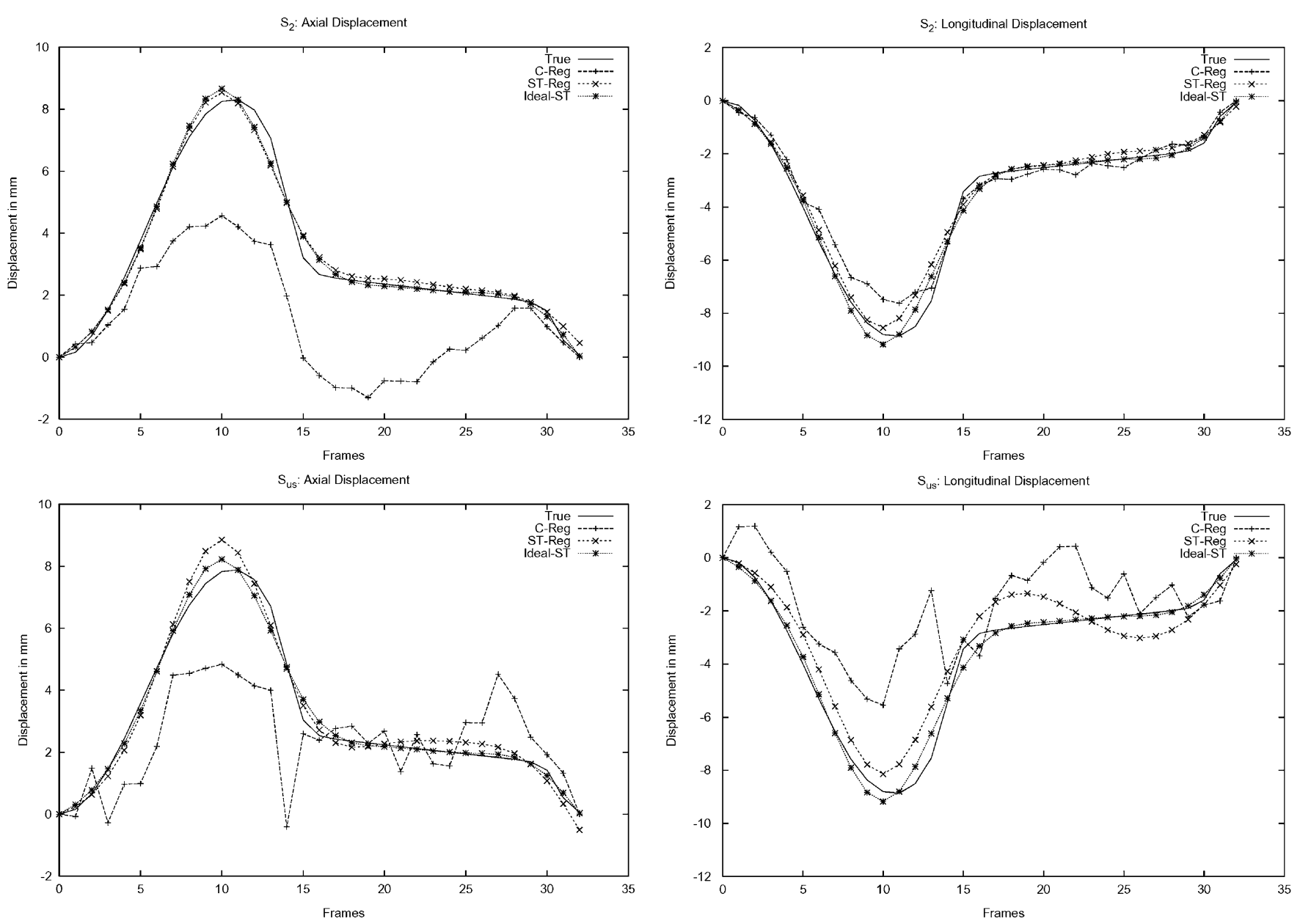

Fig. 5. Axial (left) and longitudinal (right) displacements (in millimeters) for a middle-septum point in sequences $S_{2}$ (top) and $S_{\text {us }}$ (bottom). We show the true displacement, the displacement found by the two algorithms, and the best achievable result within the frame of the motion model used in the constrained algorithm.

troduced in the C-Reg algorithm which penalizes large motions. The proposed spatio-temporal registration algorithm does not need this regularization, providing smoother movements. The improvement is clearly visible.

2) Adjusting the Knot Spacing: The knot spacing in the spatio-temporal grid influences the intrinsic resolution of the deformation that can be recovered in both time and space. When noise is present, the optimum values should be chosen as a compromise between the approximation error, which is dominant for coarser grids, and the lack of regularization for fine grids, which increases the effect of noise. The need for this compromise is particularly evident for sequence $S_{\mathrm{us}}$, as this sequence was designed to be highly decorrelated in time. In this set of experiments, we independently varied the knot spacing in space, $h$, and in time, $s$, to observe their influence.

Fig. 6 shows the geometric error for different values of the knot spacing, $h$ for a fixed value of $s=5$. The projection error ("ideal" error) decreases with the step size, $h$. However, once the true scale of the displacement is achieved, the changes become very small. Therefore, for efficiency reasons, a value of $h$ between 32 and 64 pixels should be chosen. A value of $h=32$ corresponds to $1.2 \mathrm{~cm}$ in our images, a distance similar to the thickness of the myocardium.

As expected, for each noise level there is an optimum value of $h$ that provides a sufficient level of smoothing to counteract

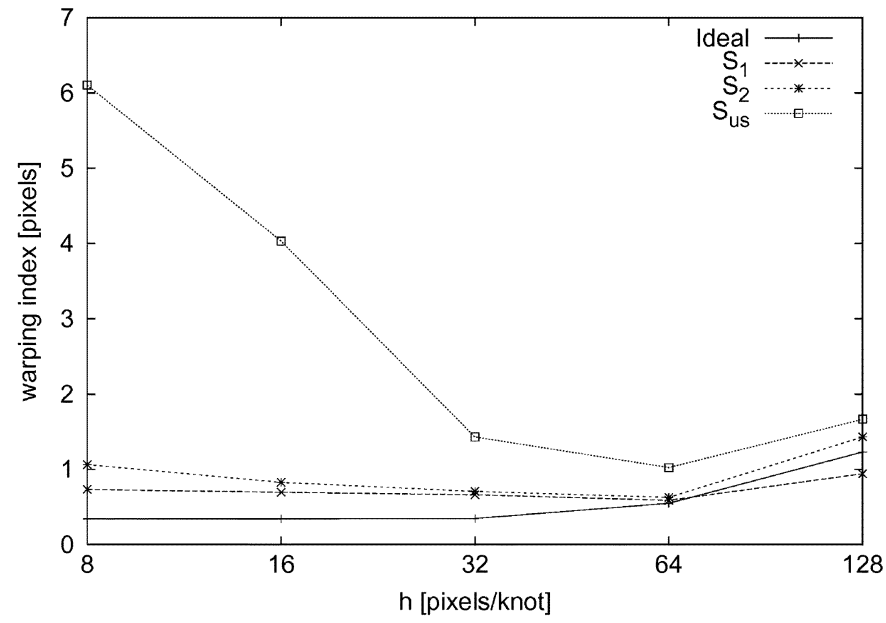

Fig. 6. The dependence of the geometrical error on the knot spacing in space $(h)$.

the noise, with sufficient flexibility in the motion model to be able to represent the deformation without too much error.

The second experiment evaluated the effect of the temporal knot spacing, $s$, for a fixed value of $h=32$. The results are summarized in Fig. 7. Observe that for no (the "ideal" case) or low noise, the error decreased as we reduced the temporal knot spacing. However, for higher noise levels, the optimum 


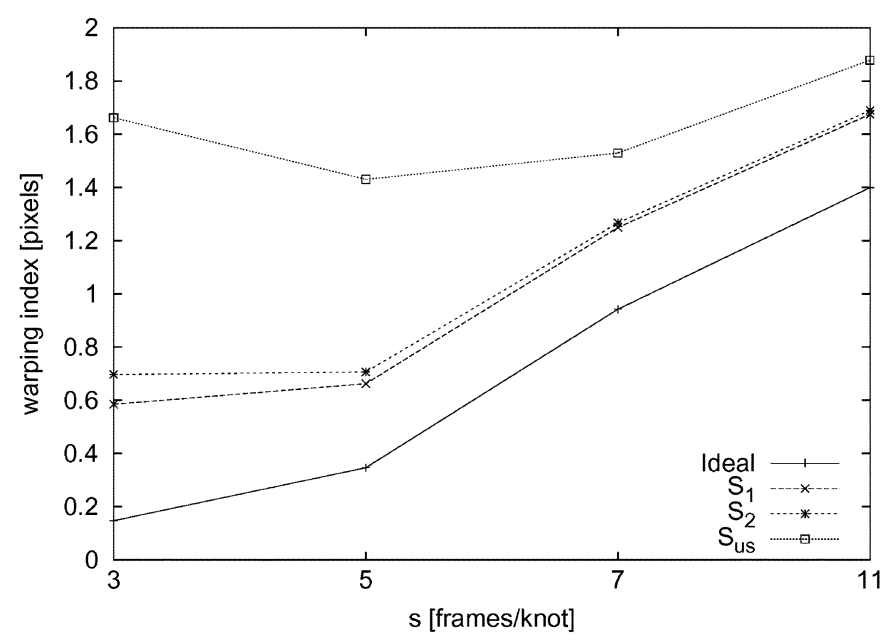

Fig. 7. Geometric error for different values of knot spacing in time $(s)$.

value was achieved for $s=5$, showing the beneficial effect of the constrained model. This was most visible for sequence $S_{\mathrm{us}}$, which had a much weaker temporal correlation than sequences $S_{1}$ and $S_{2}$.

\section{Application to Regional ANALysis of the LeFt VENTRICLE}

\section{A. Data Description and Methodology}

We now describe the use of our algorithm in a clinical setting. The assessment of myocardial wall motion and contractility is a key issue in diagnostic echocardiography, as many cardiac pathologies develop wall-motion abnormalities. Wall motion is commonly assessed qualitatively by examining the displacement and thickening of each myocardial segment. This process is usually denoted as regional analysis. The American Society of Echocardiography [47], [48] proposed a standard left ventricular division of 16 segments that have a correspondence with the irrigation areas of the main coronary arteries (Fig. 8). Despite the standardization of acquisition and scoring protocols [48], there are some inter-institutional disagreements on regional analysis interpretation [49], and quantitative methods are required to homogenize the interpretation of such studies.

In this context, we set up a small clinical test to demonstrate the applicability of our method to the quantitative regional analysis of the left ventricle. We acquired data from six healthy volunteers and six patients using a Siemens-ACUSON Sequoia ${ }^{\circledR}$ scanner. The six patients had severe wall-function abnormalities with prior infarct in the territory of the anterior descending coronary artery, and in some cases they had inferior infarcts. This damage had led to motion and contraction problems of the anterior, septal, and inferior walls. An expert performed qualitative regional analysis, grading each segment according to a standard score $(1=$ normal, $2=$ hypokinetic, and 3 = akinetic) [47]. For each subject, we analyzed two sequences: Apical two-chamber (2C) and four-chamber (4C) views, yielding 24 sequences. Our study quantified the function of the basal and mid segments for the inferior (2C view) and septal walls ( $4 \mathrm{C}$ view), a total of 48 segments. We selected these segments as they were clearly visible in all the sequences. Our evaluation focused on the systolic function using two dif-

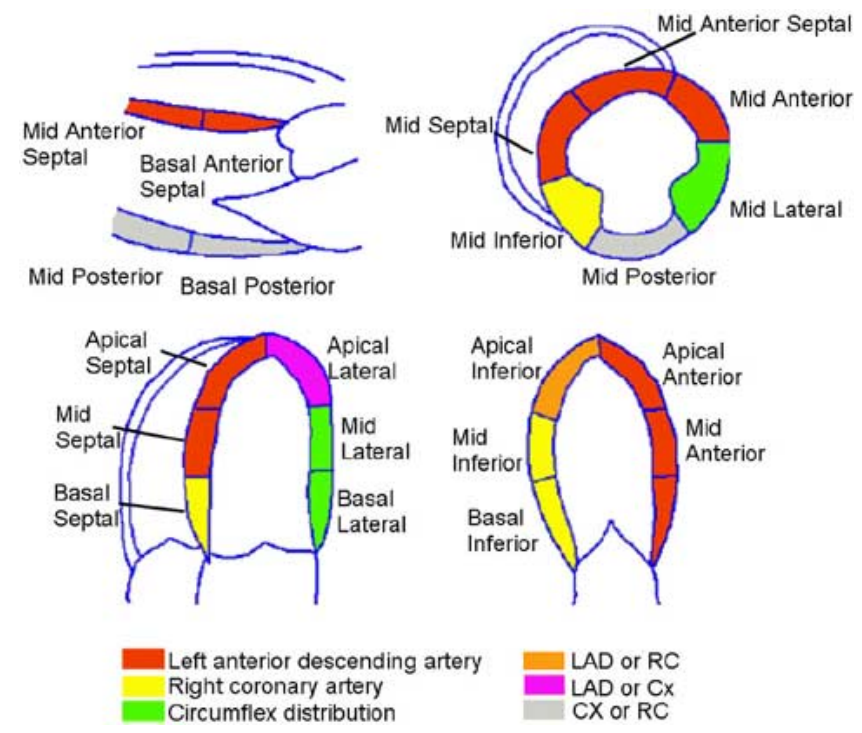

Fig. 8. Standard definition of the left ventricular 16 segments by the American Society of Echocardiography. Different textures represent the irrigation areas of the main coronary arteries.

ferent parameters: the mean displacement vector $\mathbf{u}$ and the mean local deformation $\mathbf{E}$ of the segment during systole. We selected these because they are respectively linked to the global segment motion and its active contraction. We use the displacement field that was provided by our algorithm to analyze the mean segment displacement norm $(|\mathbf{u}|)$ and its longitudinal, $\left(u_{\text {long }}\right)$ and axial $\left(u_{\mathrm{ax}}\right)$ components. Local segment deformation was quantified by estimatig the axial $\left(E_{\text {ax }}\right)$ and longitudinal $\left(E_{\text {long }}\right)$ projections of the strain tensor $(\mathbf{E})$. The strain tensor $(\mathbf{E})$ was computed analytically from the displacement field, as explained in Appendix B.

The processing of each sequence was done as follows. In the first step, we estimated the myocardial motion. Then, we defined the segments of interest, and finally, we computed the mean displacement vector and mean strain tensor for each segment. The spatio-temporal registration of all the sequences provided the myocardium displacement field. We performed all the registrations on clinical data using the optimum parameter values obtained in the simulation studies (spacing between the spatial knots was $h=32$, spacing between the temporal knots was $s=5$, a stopping optimization threshold of $\varepsilon=0.01$, and quadratic temporal and spatial B-splines were used). We manually outlined the segments of interest in the first image of each sequence. We also checked that after applying the estimated displacement the segment contours were correctly repositioned in the remaining frames of the sequence, which indicated that the recovered displacement field was consistent with the real motion. The mean systolic displacement vector was calculated by taking into account all the points enclosed in the previous definition. The longitudinal and axial components were referenced to the longitudinal axis of the left ventricle, also manually defined by the expert. For each sequence, we defined the left ventricular axis and extracted the unitary vectors along this axis, $\mathbf{v}_{\text {long }}$, and perpendicular to this axis, $\mathbf{v}_{\mathrm{ax}}$. The longitudinal and axial components were computed as projections, $u_{\mathrm{ax}}=\mathbf{u} \cdot \mathbf{v}_{\mathrm{ax}}, u_{\text {long }}=$ $\mathbf{u} \cdot \mathbf{v}_{\text {long }}, E_{\text {ax }}=\mathbf{v}_{\mathrm{ax}}^{T} \mathbf{E} \mathbf{v}_{\text {ax }}$ and $E_{\text {long }}=\mathbf{v}_{\text {long }}^{T} \mathbf{E v}_{\text {long }}$. 

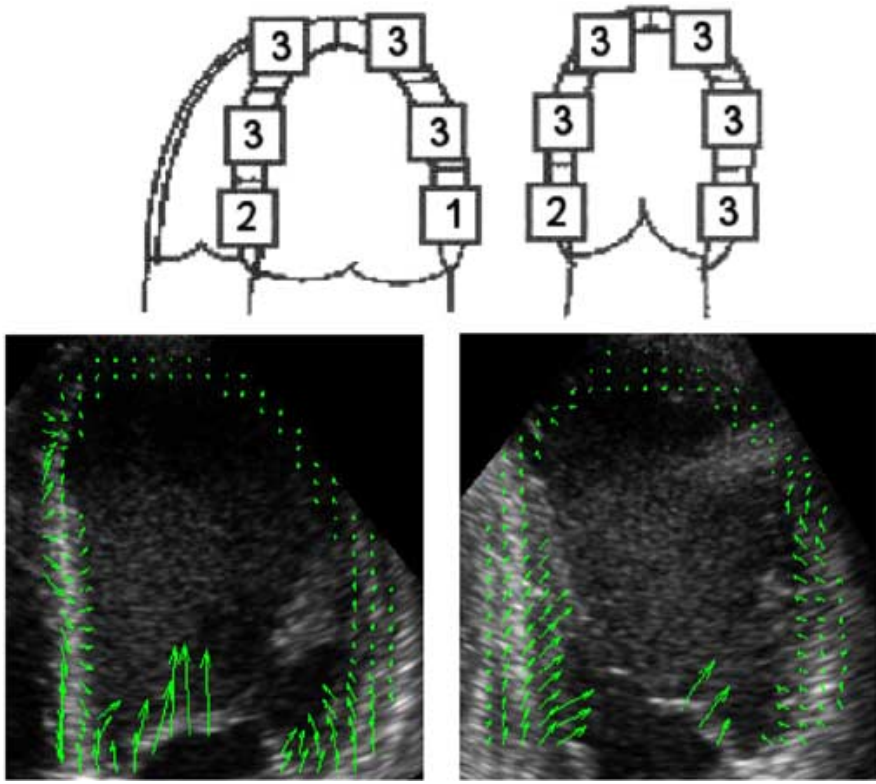

Fig. 9. Apical 4C (left) and $2 \mathrm{C}$ (right) views for a patient with an anterior acute infract. Top $=$ Expert score for each segment $(1=$ Normal, $2=$ Hypokinetic, and 3 = Akinetic). Bottom = Displacement field during systole. Akinesia is present at the anterior wall, apex, and medial septal and inferior segments. Some mobility is present in the basal septal, lateral, and inferior segments. The arrows are scaled by 1.5 times for visualization purposes.

We performed a first study to compare the displacement vector, $\mathbf{u}$, and the strain tensor, $\mathbf{E}$, in the three groups of segments: Normal, hypokinetic, and akinetic. This study considered the differences between all the segments in each group, studying a total of 24 healthy segments, 9 hypokinetic and 13 akinetic. To assess any statistical difference, we performed a one-way analysis of variance (ANOVA) followed by a post-hoc Scheffé test for multiple comparisons between groups. We report as statistically significant results with $p<0.05$.

The second analysis considered $|\mathbf{u}|$ for each individual segment independently (for example, the basal inferior) in the healthy subjects and the patients. We traced the displacement vector norm evolution, $|\mathbf{u}|$, through the systole for each segment that showed a difference between groups.

\section{B. Results}

Fig. 9 shows the displacement field at the end of systole (maximum contraction) for a patient with an anterior acute infarct in the apical $2 \mathrm{C}$ and $4 \mathrm{C}$ views. The calculated displacement field is consistent with the clinical scores. For example, notice the difference in arrow lengths between the anterior wall (left image, left wall), classified as akinetic, and the basal inferior segment, classified as hypokinetic. Fig. 10 shows the displacement field at the end of systole for a healthy volunteer.

Figs. 11 and 12 show the results of the first study that compared all the normal versus hypokinetic and akinetic segments. Considering the $\mathbf{u}$ values at the end of systole, the data in Fig. 11 represent the mean and standard deviation for $|\mathbf{u}|$ and the two components, $u_{\text {long }}$ and $u_{\text {ax }}$. ANOVA yielded a significant difference between the group means for the three parameters $\left(|\mathbf{u}|: p=0.0000 ; u_{\text {long }}: p=0.0017 ; u_{\text {ax }}: p=0.0000\right)$. The Scheffé test for $|\mathbf{u}|$ showed significant differences between each
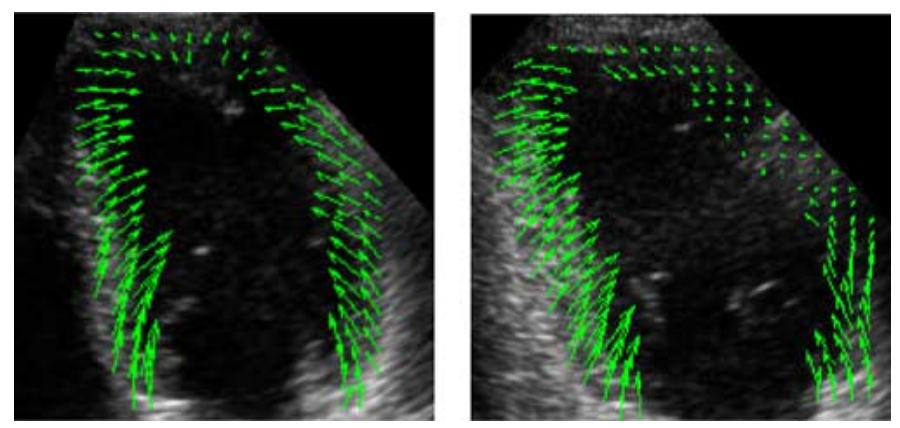

Fig. 10. The displacement field during systole for a healthy subject showing good mobility in all the segments. The arrows are scaled by 1.5 times for visualization purposes.

pair of groups (normal versus hypokinetic, $p=0.0017$; normal versus akinetic; $p=0.0000$; hypokinetic versus akinetic, $(p=$ $0.0015)$. The Scheffé test for $u_{\text {long }}$ resulted in a significant difference between only the normal and akinetic segments $(p=$ $0.0020)$ and the Scheffé test for $u_{\text {ax }}$ showed significant differences between the normal and hypokinetic $(p=0.0004)$ and the normal and akinetic segments $(p=0.0000)$. These results confirm the expected differences between the groups, especially when the global displacement is considered $(|\mathbf{u}|)$.

Fig. 12 represents the mean and standard deviation for $E_{\text {long }}$ and $E_{\text {ax }}$ considering the maximum systolic strain E. ANOVA yielded a significant difference between the group means for the two parameters $\left(E_{\text {long }}: p=0.0000 ; E_{\mathrm{ax}}: p=0.0000\right)$. The Scheffé test for $E_{\text {long }}$ showed significant differences between each pair of groups: Normal versus hypokinetic, $p=0.0482$; normal versus akinetic; $p=0.0000$; hypokinetic versus akinetic, $p=0.0293)$. The Scheffé test for $E_{\mathrm{ax}}$ showed significant differences between the normal and hypokinetic $(p=0.0004)$ and the normal and akinetic segments $(p=0.0000)$.

These results confirm the expected differences between the groups. The differences are clearly significant for all the parameters between the normal and akinetic segments. However, the change between the normal and the hypokinetic, and between the akinetic and the hypokinetic segments is not so well defined. This result is also expected, as the hypokinetic group comprises all the segments that do not move, nor contract normally, but still have some movement. Thus, it represents a group with large variance.

Table II shows the results of the second study that examined the displacement of all segments independently for healthy subjects and patients. This table shows the mean and standard deviation of $|\mathbf{u}|$ at the end of systole for each segment, and for each segment group (normal, hypokinetic, and akinetic). The results confirm the tendency observed in the first study, namely, that the segments labeled as normal have greater global motion than the hypokinetic and akinetic segments. Another observation relating to the normal segments is that the mid segments have slightly smaller global displacement values than the basal segments. This effect confirms that the longitudinal displacement decreases from base to apex. However, because of the small number of cases and the large number of categories, we did not obtain enough data to perform a meaningful statistical study.

We also studied the temporal evolution of the mean segment displacement norm, $|\mathbf{u}|$, for each segment, by comparing 


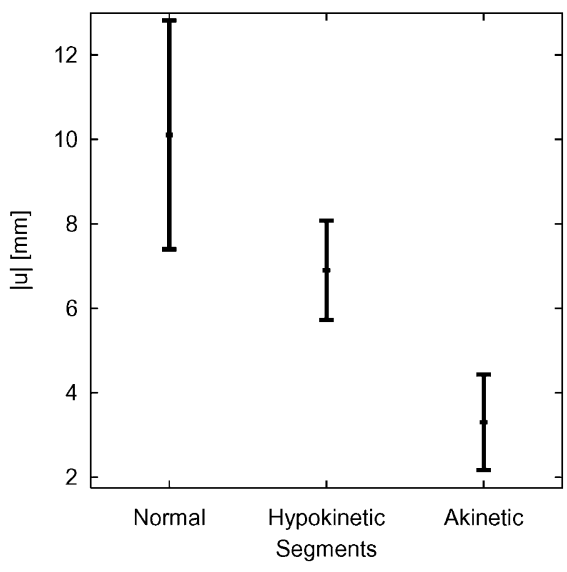

(a)

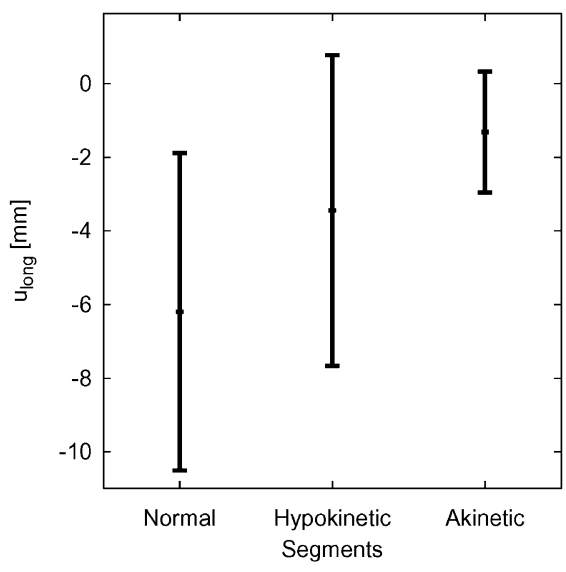

(b)

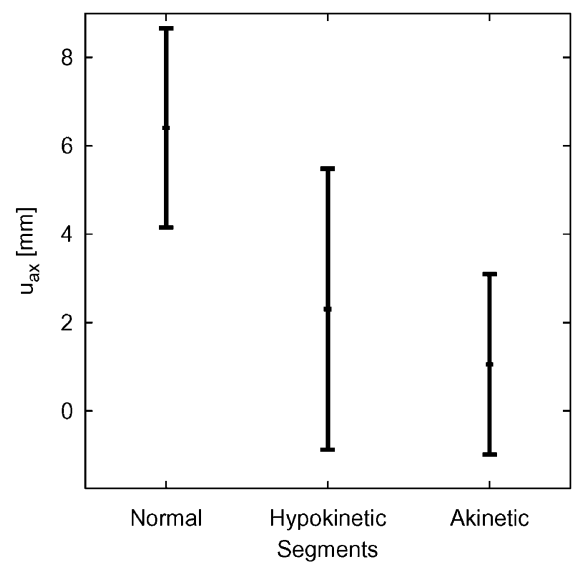

(c)

Fig. 11. (a) Mean and standard deviation for $|\mathbf{u}|$. The Scheffé test found significant differences between the three groups (normal versus hypokinetic, $p=0.0017$; normal versus akinetic, $p=0.0000$; hypokinetic versus akinetic, $p=0.0015$ ). (b) Mean and standard deviation for $u_{\text {long. Significant difference between the }}$ akinetic and normal segments $(p=0.0020)$ was observed. (c) The mean and standard deviation for $u_{\text {ax }}$. Significant difference was observed between the normal and the hypokinetic $(p=0.0004)$, and between the normal and the akinetic segments $(p=0.0000)$. Number of segments studied: $N=24$ healthy, $N=9$ hypokinetic and $N=13$ akinetic.

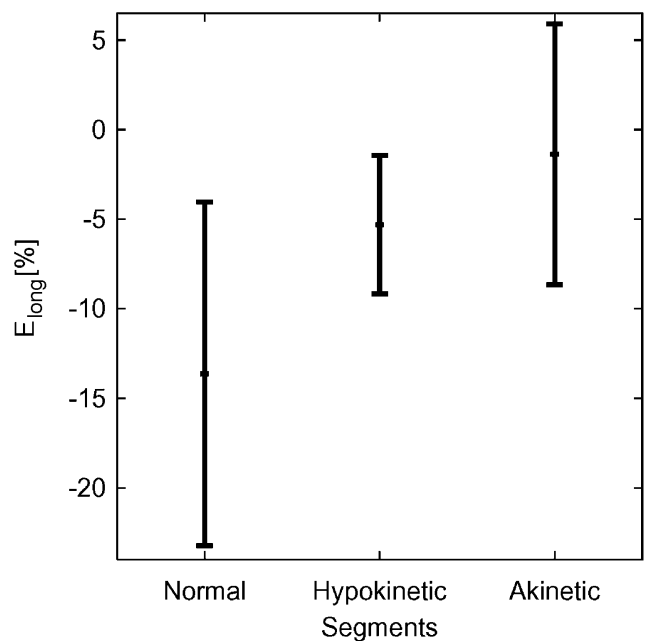

(a)

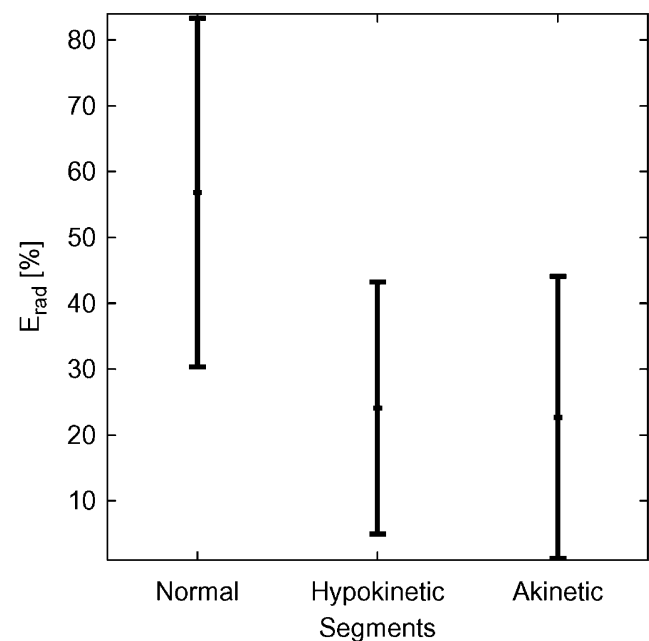

(b)

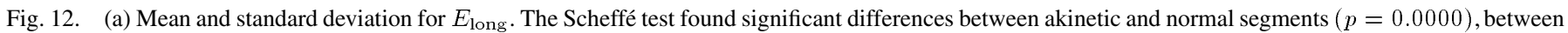
hypokinetic and normal segments $(p=0.0482)$ and between hypokinetic and akinetic $(p=0.0293)$. (b) Mean and standard deviation for $E_{\text {ax }}$. Significant difference was observed between the normal and the hypokinetic $(p=0.0004)$ and between the normal and the akinetic segments $(p=0.0000)$. Number of segments studied: $N=24$ healthy, $N=9$ hypokinetic and $N=13$ akinetic.

TABLE II

Mean and Standard DeViation of $|\mathbf{u}|($ IN Millimeters) at the END of SySTOLE FOR THE THREE SEGMENT GROUPS, STUDYING EACH SEGMENT INDEPENDENTLY. SOME COMBINATIONS DiD NOT OCCUR IN OUR DATA SET

\begin{tabular}{c||c|c|c}
\hline Segment & Normal & Hypokinetic & Akinetic \\
\hline Basal Septal & $11.63 \pm 2.87$ & $6.91 \pm 1.54$ & -- \\
& $N=6$ & $N=5$ & $N=0$ \\
\hline Mid Septal & $9.70 \pm 2.31$ & $6.60 \pm 1.07$ & $3.55 \pm 0.62$ \\
& $N=6$ & $N=2$ & $N=3$ \\
\hline Basal Inferior & $10.27 \pm 3.58$ & $7.16 \pm 0.31$ & $2.50 \pm 0.18$ \\
& $N=6$ & $N=2$ & $N=4$ \\
\hline Mid Inferior & $8.81 \pm 1.89$ & -- & $3.71 \pm 1.45$ \\
& $N=6$ & $N=0$ & $N=6$ \\
\hline
\end{tabular}

healthy volunteers and patients. Fig. 13 shows the temporal evolution during systole, $|\mathbf{u}|$, for the basal inferior segment for healthy subjects [Fig. 13(left)], and for patients [Fig. 13(right)]. Note that the basal inferior segment was hypokinetic for patients P-2 and P-4, and akinetic for the rest of the patients. The difference in temporal evolution and maximum displacement at the end of systole is noticeable when one compares the normal segments with the hypokinetic and the akinetic segments.

\section{DISCUSSION AND CONCLUSION}

In this paper, we presented a new, fully automatic procedure to compute cardiac motion from echocardiographic sequences using nonrigid registration techniques.

Our method exploits the temporal coherence of the movement, and estimates the motion field by registering the sequence to a reference frame. We used a B-spline spatio-temporal parametric model to define the displacement field. This field was expressed analytically, providing a good framework for calculating motion parameters, such as the velocity, acceleration, or 

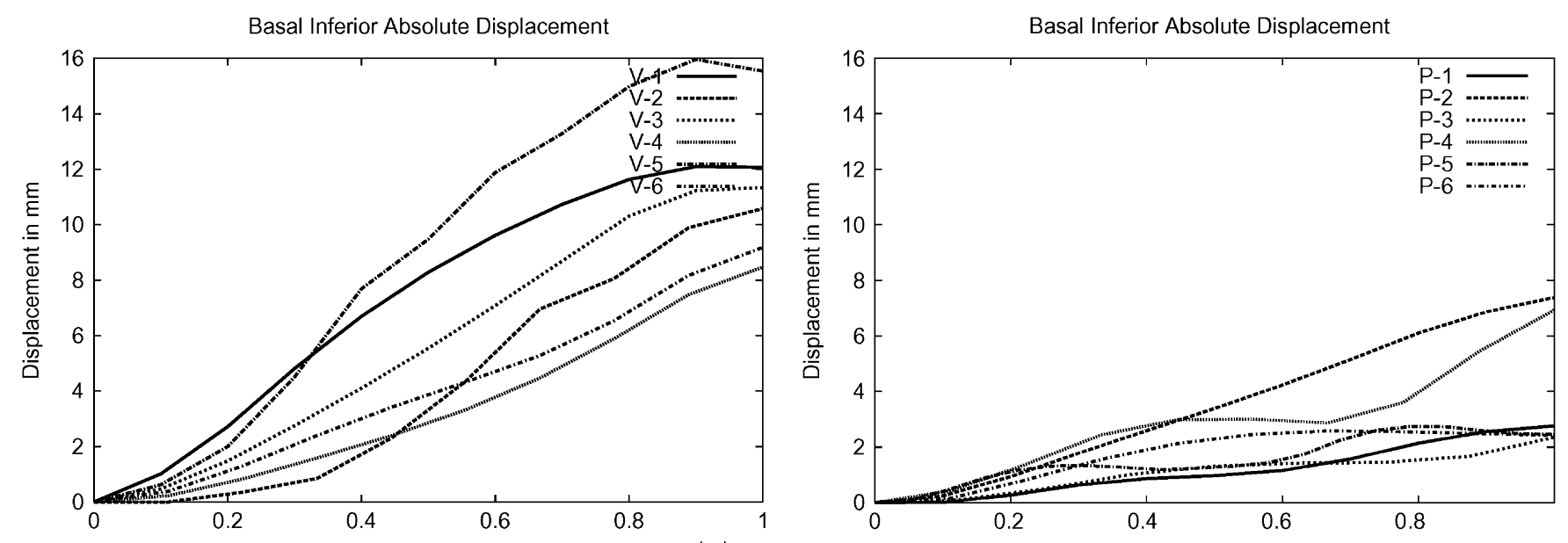

Fig. 13. Basal inferior mean absolute displacement temporal evolution $|\mathbf{u}|$ during systole for healthy subjects (left), and for patients (right). The ordinate represents incremental displacements (in millimeters) and the abscissa represents time during systole normalized to the systole duration (from 0 to 1 ).

strain. In comparison with previous approaches for motion recovery from echocardiography, which are only based on local information, our method is able to balance the weight of local and global variations.

The key methodological contributions of the present work are as follows. The first one is the specification of the problem as a global optimization over the whole sequence using as similarity measure a temporal extension of the SSD criterion. Secondly the use of a parametric spatio-temporal deformation model that we constrained adding periodicity to better represent the cyclic behavior of the cardiac motion. Another contribution is the continuous representation of sequence and deformation model, which allows for exact computation of partial derivatives. And finally, we propose a fast optimization strategy using a multiresolution approach both in the sequence and in the deformation model.

Evaluation experiments demonstrated that the proposed method is able to estimate motion accurately within 5\% of the maximum displacement for SNRs above $15 \mathrm{~dB}$, and that it can provide plausible heart motion fields from real echocardiograms. Algorithm parameters were adjusted using realistically looking simulated sequences.

We demonstrated the benefits of temporal consistency in ultrasound motion recovery by comparing the data with those using a previous algorithm [37] based on nonrigid registration of independent consecutive image pairs.

The accuracy of the current method may be affected by the intrinsic limitations of the echocardiographic imaging modality. The most important of these are the partially decorrelated speckle, the out-of-plane motion, the attenuation, and the independent movement of intraventricular structures (e.g., valves and papillary muscles). The results provided suggest that our approach works even in the presence of these effects. However, the large attenuation in some myocardial regions (e.g., lateral wall in apical 4C views) could prevent its use in these areas. The out-of-plane motion causes a myocardial texture change which may also affect the accuracy of the local motion estimates. This effect is reduced by the spatial and temporal consistency provided by the spatio-temporal parametric model. Intraventricular structures appearing and disappearing from the view-plane may also be a cause of error, partially compensated by temporal smoothness. In areas where with low signal intensity (e.g., the bloodpool) the estimated motion maybe somewhat erratic. However, this effect hardly interferes with the myocardial tracking as there is much more energy and temporal coherence within the myocardium. If this effect appears to be problematic in an extensive evaluation, there are two different ways to deal with it: Adding regularization to the registration process, or including a mask to select only the myocardium.

The results obtained with real data, from normal subjects and from patients, suggest the clinical applicability to the regional analysis of the left ventricle. Both displacement and strain results show significant differences between normal and pathological segments. Displacement and strain values are consistent with those previously published and obtained with Doppler derived techniques [50]-[52], and Tagged MR data [53], [54]. These results reveal the potential of this technique to provide a new method to assess myocardial motion from echocardiographic data, overcoming the limitations of the Doppler techniques. This preliminary clinical evaluation encourages further research on the use of the proposed method to derive quantitative parameters to indicate the presence of ischemic disease. This clinical validation should also consider whether the variability in the definition of long axis by the user has any influence on the results.

It may also be interesting to test the proposed algorithm in other medical imaging contexts. Potential applications include motion estimation from other cardiac imaging modalities, cardiac sequence segmentation guided by registration, and coding/compression of movement components.

\section{APPENDIX}

\section{A. Simulated Cardiac Motion}

Our simulated cardiac motion was derived to mimic the movement found in a $4 \mathrm{C}$ sequence of a normal volunteer. We describe this model analytically, to be a separable model in time and space

$$
\mathbf{g}_{0}(t, \mathbf{x})=\zeta(t) \xi(\mathbf{x})=\zeta(t)\left[\begin{array}{c}
a_{x} \sin \frac{\pi\left(x_{\mathrm{ax}}-x\right)}{2\left|x_{\mathrm{endo}}-x_{\mathrm{ax}}\right|} \\
a_{y} \frac{\left|y_{\text {apex }}-y\right|}{\left|y_{\text {apex }}-y_{\text {valve }}\right|}
\end{array}\right]
$$




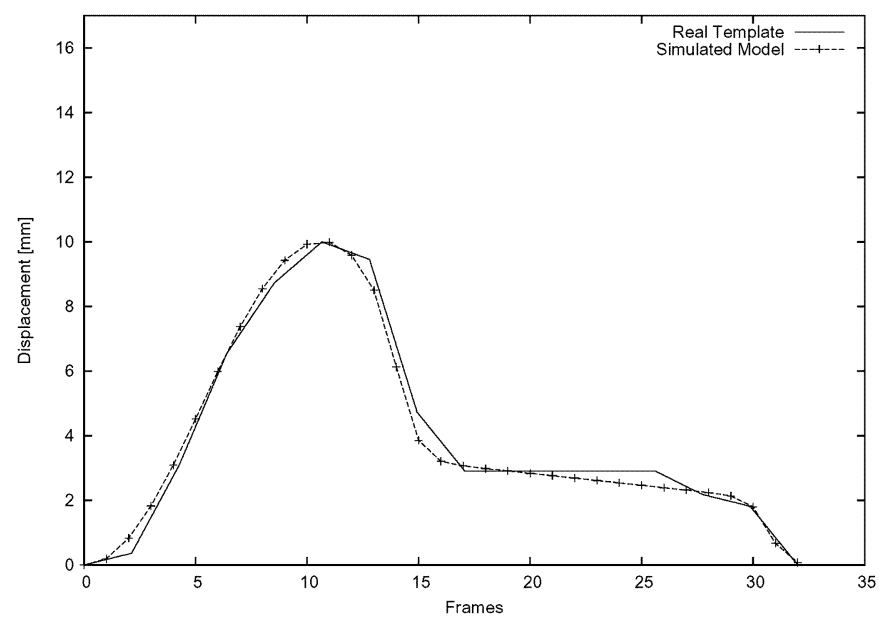

Fig. 14. A simulated temporal function, $\zeta(t)$, and the template extracted from the real sequence.

where $\xi(\mathbf{x})$ is the spatial dependence term, $\zeta(t)$ is the temporal term, $t$ is the frame time, and $\mathbf{x}$ is the spatial coordinate. The spatial dependence is also separated along longitudinal $(y)$ and axial components $(x)^{1}(12)$ specifies that the axial component, $x$, is maximum for the endocardium, $x_{\mathrm{endo}}$, and symmetric with respect to the longitudinal axis of the left ventricle, $x_{\mathrm{ax}}$, which is oriented vertically; i.e., parallel to the $y$ axis. The longitudinal displacement, $y$, is maximum for the insertions of the mitral valve, $y_{\text {valve }}$, and zero for the apex, $y_{\text {apex }}$. Coordinate values and maximum magnitudes were taken from a real sequence used as a template ( $a_{x}=16$ and $a_{y}=11$ pixels).

This model simulates the regional dependence of healthy myocardial motion that is linked to the muscle structure and contraction process [55], [56]. The maximum axial displacement during systole occurs at the endocardium, and is smaller toward the epicardium, leading to a transmural gradient that produces wall thickening. Similarly, longitudinal displacement decreases from base to apex, creating a spatial gradient in this direction that represents the myocardial longitudinal function.

The temporal term, $\zeta(t)$, was also defined using a real sequence as a template. We extracted the average temporal evolution from several points in the myocardium, and an analytical expression was fitted to the extracted function (Fig. 14). We defined $\zeta(t)$ in a piece-wise fashion using a continuous pulse function, $\sqcap\left(t_{i}, t_{i+1}\right)$

$$
\zeta(t)=\sqcap\left(t_{0}, t_{1}\right) \sin ^{2} \frac{\pi t}{\frac{2}{3} T}+\sqcap\left(t_{1}, t_{2}\right)(a+b * t)
$$

where $t_{0}=0.0 \cdot T, t_{1}=0.446 \cdot T, t_{2}=0.960 \cdot T, a=0.431, b=$ -0.007 , and $T=33$ image frames.

$\sqcap\left(t_{i}, t_{i+1}\right)=\frac{1}{4}\left(1+\tanh \left(\alpha\left(t-t_{i}\right)\right)\right)\left(1+\tanh \left(\alpha\left(t_{i-1}-t\right)\right)\right)$.

where $\alpha=1.136$.

${ }^{1}$ In the context of this section we use $(x, y)$ instead of $\left(x_{1}, x_{2}\right)$ for clarity.

\section{B. Strain Calculation}

The movement of a nonrigid homogeneous body is usually composed of changes in shape, size, and position [57]. It can be decomposed into a rigid and a nonrigid component. Given a nonrigid body $B_{0}$, with a particle at position $\mathbf{X}$, moved and deformed into body $B_{t}$, with a new position of the same particle at $\mathbf{x}$, we define the deformation gradient tensor as

$$
\mathbf{F}=\nabla_{\mathbf{X}}(\mathbf{x}) \quad F_{i j}=\frac{\partial x_{i}}{\partial X_{j}} \quad F_{i j}=\frac{\partial u_{i}}{\partial X_{j}}+1
$$

where $u=\mathbf{x}-\mathbf{X}$ is the displacement of the given particle.

$\mathbf{F}$ represents the body variations in shape, size and position. It can be decomposed in two matrices $\mathbf{F}=\mathbf{R U}$, where $\mathbf{R}$ is the rotation matrix y $\mathbf{U}$ is the right stretch tensor. This decomposition is quite complex; therefore, the Cauchy-Green tensor is also defined as $\mathbf{C}=\mathbf{F}^{T} \mathbf{F}=U^{2}$. This formulation allows the definition of the Green-Lagrange strain tensor as

$$
\mathbf{E}=\frac{1}{2}(\mathbf{C}-\mathbf{I})=\frac{1}{2}\left(\mathbf{F}^{T} \mathbf{F}-\mathbf{I}\right)
$$

where $\mathbf{E}=0$ when no deformation exists.

Given the dense displacement field $\mathbf{g}(t, \mathbf{x})$ introduced in the Section II and taking into account (15), we can calculate $\mathbf{F}$ in the bidimensional case as

$$
\mathbf{F}=\nabla_{\mathbf{x}} \mathbf{g}+I=\left[\begin{array}{ll}
\frac{\partial g_{1}}{\partial x_{1}} & \frac{\partial g_{1}}{\partial x_{2}} \\
\frac{\partial g_{2}}{\partial x_{1}} & \frac{\partial g_{2}}{\partial x_{2}}
\end{array}\right]+I
$$

The Green-Lagrange strain tensor $\mathbf{E}$ is then easily computed from (16). Components $\left(\partial g_{i}\right) /\left(\partial x_{j}\right)$ can be calculated analytically in the case of our deformation model (4) as it is defined through Bspline functions. The deformation tensor is then projected onto the directions of interest to calculate the deformation in a determined direction.

\section{ACKNOWLEDGMENT}

The authors wish to acknowledge the contribution of Dr. M. A. García Fernández and Dr. E. Pérez David for their support and fruitful discussions during the clinical trials.

\section{REFERENCES}

[1] P. Clarysse, C. Basset, L. Khouas, P. Croisille, D. Friboulet, C. Odet, and I. Magnin, "Two-dimensional spatial and temporal displacement and deformation field fitting from cardiac magnetic resonance tagging," Med. Image Anal., vol. 4, no. 4, pp. 253-268, 2000.

[2] J. C. McEachen, A. Nehorai, and J. S. Duncan, "Multiframe temporal estimation of cardiac nonrigid motion," IEEE Trans. Med. Imag., vol. 9, no. 4, pp. 651-664, Apr. 2000.

[3] P. Shi, A. J. Sinusas, R. T. Constable, and J. S. Duncan, "Volumetric deformation analysis using mechanics-based data fusion: Applications in cardiac motion recovery," Int. J. Comput. Vision, vol. 35, no. 1, pp. 87-107, 1999

[4] J. Declerck, J. Feldmar, and N. Ayache, "Defininion of a 4D continuous planispheric transformation for the tracking and the analysis of left-ventricle motion," Med. Image Anal., vol. 2, no. 2, pp. 197-213, 1998. 
[5] C. Nastar and N. Ayache, "Frequency-based nonrigid motion analysis application to four dimensional medical images," IEEE Trans. Pattern Anal. Mach. Intell., vol. 18, no. 11, pp. 1067-1079, Nov. 1996.

[6] P. Radeva, A. Amini, and J. Huang, "Deformable B-solids and implicit snakes for 3D localization and tracking of SPAMM-MRI data," Comput. Vis. Image Understanding, vol. 66, pp. 163-178, May 1997.

[7] J.-P. Thirion and S. Benayoun, "Myotrack: A 3D deformation field method to measure cardiac motion from gated SPECT," in Lecture Notes in Computer Science, S. L. Delp, A. M. DiGioia, and B. Jaramaz, Eds. Berlin, Germany: Springer Verlag, Oct. 2000, vol. 1935, Proc. MICCAI 2000, pp. 697-706.

[8] J. Declerck, J. Feldmar, M. L. Goris, and F. Betting, "Automatic registration and alignment on a template of cardiac stress and rest reoriented spect images," IEEE Trans. Med. Imag., vol. 16, no. 6, pp. 727-737, Dec. 1999.

[9] G. J. Klein and R. H. Huesman, "Four-dimensional processing of deformable cardiac PET data," Med. Image Anal., vol. 6, pp. 29-46, 2002.

[10] J.-M. Gorce, D. Fibroulet, and I. Magnin, "Estimation of three dimensional cardiac velocity fields: Assessment of a diferential method and application to three-dimensional CT data," Med. Image Anal., vol. 1, no. 3, pp. 245-261, 1996

[11] K. A. Bartels, A. C. Bovik, and C. E. Griffin, "Spatio-temporal tracking of material shape change via multi-dimensional splines," in Proc. IEEE Workshop Biomedical Image Analysis, Jun. 1994, pp. 110-116.

[12] R. Szeliski and J. Coughlan, "Spline-based image registration," Int. J. Comput. Vision, vol. 22, pp. 199-218, 1997.

[13] Y. Caspi and M. Irani, "Spatio-temporal alignment of sequences," IEEE Trans. Pattern Anal. Mach. Intell., vol. 24, no. 11, pp. 1409-1424, Nov. 2002.

[14] T. Makela, P. Clarysse, O. Sipila, N. Pauna, Q. C. Pham, T. Katila, and I. Magnin, "A review of cardiac image registration methods," IEEE Trans. Med. Imag., vol. 21, no. 9, pp. 1011-1021, Sep. 2002.

[15] J. A. Noble, D. Dawson, J. Lindner, J. Sklenar, and S. Kaul, "Automated, nonrigid alignment of clinical myocardial contrast echocardiography image sequences: Comparison with manual alignment," Ultrasound Med. Biol., vol. 28, no. 1, pp. 115-123, 2002.

[16] J. Huang, D. Abendschein, V. Davila-Roman, and A. Amini, "Spatio-temporal tracking of myocardial deformations with a 4-D B-spline model from tagged MRI," IEEE Trans. Med. Imag., vol. 18, no. 10 , pp. $957-972$, Oct. 1999.

[17] C. Ozturk and E. R. McVeigh, "Four-dimensional B-spline based motion analysis of tagged MR images: Introduction and in vivo validation," Phys. Med. Biol., vol. 45, no. 6, pp. 1683-1702, 2000.

[18] D. Rueckert, M. Lorenzo-Valdes, R. Chandrashekara, G. Sanchez-Ortiz, and R. Mohiaddin, "Non-rigid registration of cardiac mr: Application to motion modeling and atlas-based segmentation," in Proc. 1st 2002 IEEE Int. Symp. Biomedical Imaging: Macro to Nano (ISBI'02), vol. II, Washington, DC, Jul. 2002, pp. 481-484.

[19] R. Chandrashekara, R. H. Mohiaddin, and D. Rueckert, "Analysis of myocardial motion in tagged MR images using nonrigid image registration," Proc. SPIE (Medical Imaging 2002: Image Processing), pp. 1168-1179, Feb. 2002.

[20] J. Meunier and M. Bertrand, "Echographic image mean gray level changes with tissue dynamics: A system-based model study," IEEE Trans. Biomed. Eng., vol. 42, no. 4, pp. 403-410, Apr. 1995.

[21] _ - "Ultrasonic texture motion analysis: Theory and simulation," IEEE Trans. Med. Imag., vol. 14, pp. 293-300, Jun. 1995.

[22] A. Giachetti, "On-line analysis of echocardigraphic imge sequences," Med. Image Anal., vol. 2, no. 3, pp. 261-284, 1998.

[23] I. Mikiæ, S. Krucinski, and J. D. Thomas, "Segmentation and tracking in echocardiographic sequences: Active contours guided by optical flow estimates," IEEE Trans. Med. Imag., vol. 17, no. 2, pp. 274-284, Apr. 1998.

[24] G. Jacob, A. J. Noble, C. Behrenbruch, A. D. Kelion, and A. P. Banning, "A shape-space-based approach to tracking myocardial borders and quantifying regional left-ventricular function applied in echocardiography," IEEE Trans. Med. Imag., vol. 21, no. 3, pp. 226-238, Mar. 2002.

[25] J. Bosch, S. Mitchell, B. Lelieveldt, O. Nijland, F. Kamp, M. Sonka, and J. Reiber, "Automatic segmentation of echocardiographic sequences by active appearance motion models," IEEE Trans. Med. Imag., vol. 11, no. 11, pp. 1374-1383, Nov. 2002.

[26] G. Mailloux, F. Langlois, P. Simard, and M. Bertrand, "Restoration of the velocity field of the heart from two dimensional echocardiograms," IEEE Trans. Med. Imag., vol. 8, no. 2, pp. 143-153, Jun. 1989.

[27] Y. Chunke, K. Terada, and S. Oe, "Motion analysis of echocardiograph using optical flow method," in Proc. IEEE Int. Conf. Systems, Man and Cybernetics, 1996, pp. 672-677.
[28] P. Baraldi, A. Sarti, C. Lamberti, A. Prandini, and F. Sgallari, "Evaluation of differential optical flow techniques on synthesized echo images," IEEE Trans. Med. Imag., vol. 43, no. 3, pp. 259-272, Mar 1996.

[29] B. Cohen and I. Dinstein, "New maximum likelihood motion estimation schemes for noisy ultrasound images," Pattern Recogn., vol. 35, no. 2, pp. $455-463,2002$.

[30] D. Boukerroui, J. Brady, and J. Noble, "Velocity estimation in ultrasound images: A block matching approach," in Proc. 18th Information Processing in Medical Imaging (IPMI), Ambleside, UK, 2003, pp. 586-598.

[31] M. Sühling, M. Arigovindan, C. Jansen, P. Hunziker, and M. Unser, "Myocardial motion analysis from B-mode echocardiograms," IEEE Trans. Image Process., vol. 14, no. 4, pp. 525-536, Apr. 2005.

[32] K. Kaluzynski, X. Chen, S. Emelianov, A. Skovoroda, and M. O'Donnell, "Strain rate imaging using two-dimensional speckle tracking," IEEE Trans. Ultrason., Ferroelec, Freq. Contr., vol. 48, no. 4, pp. 1111-1123, Jul. 2001.

[33] I. Hein and W. O'Brien, "Current time-domain methods for assessing tissue motion by analysis from reflected ultrasound echoes-A review," IEEE Trans. Ultrason., Ferroelec, Freq. Contr., vol. 40, no. 2, pp. 84-102, Mar. 1993.

[34] E. Konofagou and J. Ophir, "A new elastographic method for estimation and imaging of lateral displacements, lateral strains, corrected axial strains and poisson's ratios in tissues," Ultrasound Med. Biol., vol. 24, no. 8, pp. 1183-99, 1998

[35] J. D'hooge, E. Konofagou, F. Jamal, A. Heimdal, L. Barrios, B. Bijnens, J. Thoen, F. Van de Werf, G. Sutherland, and P. Suetens, "Twodimensional ultrasonic strain rate measurement of the human heart in vivo," IEEE Trans. Ultrason., Ferroelec, Freq. Contr., vol. 49, no. 2, pp. 281-286, Feb. 2002

[36] F. Yeung, S. F. Levinson, D. Fu, and K. J. Parker, "Feature-adaptive motion tracking of ultrasound image sequences using a deformable mesh," IEEE Trans. Med. Imag., vol. 17, pp. 945-956, Dec. 1998.

[37] M. Ledesma-Carbayo, J. Kybic, M. Desco, A. Santos, and M. Unser, "Cardiac motion analysis from ultrasound sequences using nonrigid registration," in Lecture Notes in Computer Science, W. Niessen and M. Viergever, Eds. Berlin, Germany: Springer-Verlag, Oct. 2001, vol. 2208, MICCAI 2001, pp. 889-896.

[38] J. Kybic and M. Unser, "Fast parametric elastic image registration," IEEE Trans. Image Process., vol. 12, no. 11, pp. 1427-1442, Nov. 2003.

[39] M. Unser, A. Aldroubi, and M. Eden, "Fast B-spline transforms for continuous image representation and interpolation," IEEE Trans. Pattern Anal. Mach. Intell., vol. 13, no. 3, pp. 277-285, Mar. 1991.

[40] D. Rueckert, L. Sonoda, C. Hayes, D. Hill, M. Leach, and D. Hawkes, "Nonrigid registration using free-form deformations: Application to breast mr images," IEEE Trans. Med. Imag., vol. 18, no. 8, pp. 712-721, Aug. 1999.

[41] M. Ledesma-Carbayo, "Cardiac motion detection using elastic registration," Ph.D. thesis, Universidad Politécnica de Madrid, Madrid, Spain, 2003.

[42] M. Unser, A. Aldroubi, and M. Eden, "The $L_{2}$-polynomial spline pyramid," IEEE Trans. Pattern Anal. Mach. Intell., vol. 15, no. 4, pp. 364-379, Apr. 1993.

[43] J. Jensen, "Field: A program for simulating ultrasound systems," Med. Biol. Eng. Comput. (10th Nordic-Baltic Conference on Biomedical Imaging Published in Medical \& Biological Engineering \& Computing), vol. 34, pp. 546-556, 1996.

[44] J. Jensen and N. B. Svendsen, "Calculation of pressure fields from arbitrarily shaped, apodized, and excited ultrasound transducers," IEEE Trans. Ultrason., Ferroelec, Freq. Contr., vol. 39, no. 2, pp. 262-267, Mar. 1992

[45] J. A. Jensen and P. Munk, "Computer phantoms for simulating ultrasound B-mode and cfm images," in Proc. 23rd Acoustical Imaging Symposium, Boston, MA, 1997.

[46] P. Thévenaz, U. E. Ruttimann, and M. Unser, "A pyramid approach to subpixel registration based on intensity," IEEE Trans. Image Process. vol. 7, no. 1, pp. 1-15, Jan. 1998.

[47] N. Schiller, P. Shah, and M. Crawford, "American Society of Echocardiography committee on standards, subcommittee on quantitation of two-dimensional echocardiograms: Recommendations for quantitation of the left ventricle by two-dimensional echocardiography," J. Am. Soc. Echocardiol., vol. 17, pp. 358-367, Dec. 1989.

[48] N. B. Schiller, "Two-dimensional echocardiographic determination of left ventricular volume, systolic function, and mass. Summary and discussion of the 1989 recommendations of the American society of echocardiography," Circulation, vol. 84, no. 3 Suppl, pp. 1180-1187, 1991. 
[49] R. Hoffmann, H. Lethen, T. Marwick, F. A. Flachskampf, P. Hanrath, M. Arnese, P. Fioretti, A. Pingitore, E. Picano, T. Buck, and T. Erbel, "Analysis of interinstitutional observer agreement in interpretation of dobutamine stress echocardiograms," J. Am. Coll. Cardiol., vol. 27, pp. 330-336, Feb. 1996.

[50] N. Andersen and S. Poulsen, "Evaluation of the longitudinal contraction of the left ventricle in normal subjects by Doppler tissue tracing and strain rate," J. Am. Soc. Echocardiogr., vol. 16, pp. 716-723, Jul. 2003.

[51] T. Kukulski, F. Jamal, L. Herbots, J. D'hooge, B. Bijnens, L. Hatle, I. De Scheerder, and G. Sutherland, "Identification of acutely ischemic myocardium using ultrasonic strain measurements. A clinical study in patients undergoing coronary angioplasty.," J. Am. Coll. Cardiol, vol. 41, no. 5, pp. 810-819, 2003.

[52] M. Kowalski, T. Kukulski, F. Jamal, J. D'hooge, F. Weidemann, F. Rademakers, B. Bijnens, L. Hatle, and G. Sutherland, "Can natural strain and strain rate quantify regional myocardial deformation? A study in healthy subjects," Ultrasound Med. Biol., vol. 27, no. 8, pp. 1087-1097, 2001.
[53] L. Herbots, F. Maes, J. D’hooge, P. Claus, S. Dymarkowski, P. Mertens, L. Mortelmans, B. Bijnens, J. Bogaert, F. E. Rademakers, and G. R. Sutherland, "Quantifying myocardial deformation throughout the cardiac cycle: A comparison of ultrasound strain rate, grey-scale m-mode and magnetic resonance imaging," Ultrasound Med. Biol., vol. 30, no. 5, pp. 591-598, 2004.

[54] C. Moore, C. Lugo-Olivieri, E. McVeigh, and E. Zerhouni, "Three-dimensional systolic strain patterns in the normal human left ventricle: Characterization with tagged MR imaging.," Radiology, vol. 214, no. 2, pp. 453-466, 2000.

[55] J. G. Dumesnil, R. M. Shoucri, J. L. Laurenceau, and J. Turcot, "A mathematical model of the dynamic geometry of the intact left ventricle and its application to clinical data," Circulation, vol. 59, no. 5, pp. 1024-1034, 1979.

[56] D. Gibson, "Regional left ventricular wall motion," in Cardiac Ultrasound, J. R. Roelandt, G. R. Sutherland, and S. Iliceto, Eds. Edinburgh, U.K.: Churchill Livingstone, Oct. 1993, pp. 241-51.

[57] A. J. M. Spencer, Continuum Mechanics. London, U.K.: Longman Group Limited, 1980. 